\title{
Electromagnetic Fields due to an Electron Avalanche
}

\author{
Debasish Nath
}

\begin{abstract}
In electric power system and electronic system, local partial breakdown of air in the form of corona, partial discharge and ESD are a common occurrence. These processes are known to be sources of electromagnetic fields. The electromagnetic fields due to corona on overhead line conductors can be a source of electromagnetic noise over a wide frequency range which may lead to EMI. In case of partial discharge, the EM fields and especially the magnetic field can be employed to detect and possibly locate them inside apparatus. The fields due to ESD assume importance in designing suitable protection systems. Therefore, there is a need to relate the electromagnetic field produced by discharge phenomena to the physical ionization process which constitutes the discharge. The basic mechanism behind naturally initiated electrical discharges is the electron avalanche and the total electric field produced by it has been derived and validated in literature. The next step in this direction would be to obtain the magnetic field produced by an electron avalanche. This is taken up in the present work. An expression for the complete magnetic field i.e., the near and far field components are derived starting from the Leinard-Weichert potentials. The electron avalanche is modelled according to the Townsend theory of ionization. The derived expression is validated with the numerical solution of Maxwell's curl equation for electric field or Faraday's law. A simplified procedure to estimate the field due to discharge process is discussed.
\end{abstract}

\section{Index Terms - Corona, Partial Discharge (PD), Electrostatic Discharge (ESD),} Electromagnetic Field.

\section{INTRODUCTION}

In electrical power systems, localized electrical breakdown of air is a common occurrence. These include corona on overhead transmission line conductors and Partial Discharge (PD) inside High Voltage (HV) apparatus. In addition, Electrostatic Discharge (ESD) can induce disturbances leading to maloperations in large power systems [1]. ESD also is an important factor in designing the protection in electronic circuits. This has become more challenging in recent times due to increasing signal frequency, shrinking of electronic device feature size and increase in system-on-a-chip (SoC) environments [2]. Owing to their importance, significant work has been carried out to study corona, partial discharge as well as ESD in literature.

Corona on line conductors can lead to Electromagnetic Interference (EMI) with communication systems, radio and television receivers and neighbouring electrical and electronic circuits. Due to the growing importance of EMI in the present times, considerable efforts have been directed towards estimating the EMI produced by overhead lines under corona. A number of methods have been developed for this purpose and elaborate discussions on them can be found in $[3,4,5]$.

The aforesaid methods however, attempts to quantify, only the EMI produced by the corona current [5]. However, additionally, the ionization activity associated with corona can produce its own electromagnetic fields [4]. As a result, relating the physical ionization process to the electromagnetic fields produced by it assumes importance in the present times, when EMI/EMC (Electromagnetic Compatibility) is of growing concern.

Similarly, in the past two decades, detection and location of PD in GIS equipment and Transformers have been studied using approaches based on acoustic and Electromagnetic (EM) waves $[6,7,8,9]$. Detection by electrical methods in which the EM wave produced by the physical phenomenon of PD is sensed using UHF sensors or RF sensors is preferred since they provide information relevant to the physics of $\mathrm{PD}$ phenomenon $[7,9]$. Clearly, in understanding the physics of PD phenomenon and therefore in its modelling and simulation and possibly in measurement approaches, relating the physical ionization process in PD to the electromagnetic fields produced by it becomes necessary.

The fields generated by ESD can not only interfere with the operations of electronic devices and systems, but can also lead to damages (irreversible breakdown) of electronic devices [10]. As a result, in the design of ESD protection systems, knowledge of the fields produced by ESD process assumes importance.

Therefore, for various purposes, there seems to be a need to relate the physical ionization (discharge) process to the electromagnetic fields produced (as seen from the examples of corona, PD and ESD). However, literature survey indicates that work in this direction is still in very early stage. In a relatively recent literature, only the radiation (far field) component of the electric field due to a single electron avalanche, which is the 
basic mechanism behind most atmospheric discharge processes, as well as corona, PD and ESD has been studied [11].

In a more recent work, the complete expression of the electric field which includes all the components (near field and far or radiation field) for a single electron avalanche has been derived and validated [12]. However, the magnetic field due to an electron avalanche would be required to study the EM radiation and wave propagation properties. It is worthwhile to mention that in a relatively recent literature [7] it has been shown that the magnetic field due to PD can propagate through the metal tank of a transformer and be detected with RF sensor at the outer surface. Therefore, an expression for the magnetic field due to the ionization process would be extremely useful in understanding and analyzing the related effects. This forms the objective of the present work. Finally, along similar lines as has been followed in literature [11], the magnetic field from physical discharge is estimated from the results of a single avalanche.

An expression for the magnetic field due to an electron avalanche is derived starting from the Leinard-Weichert potentials and validated with the numerical solution of Maxwell's equation. The expression is valid even at relativistic speeds and therefore can be employed to study relativistic avalanches which are found during gamma ray bursts from thunderclouds [13]. The electron avalanche is modelled in terms of the well-known Townsend theory of ionization and is discussed in the following.

\section{MODELLING AN ELECTRON AVALANCHE}

The Townsend theory of ionization has been routinely employed in literature to model an electron avalanche $[11,14,15$, 16]. The same model is used in the present work with the important assumptions stated as follows:

i). Free electrons produced by external means when sufficiently accelerated by the background electric field, results in an exponential growth in the number of free electrons. i.e., single electron can lead to

$q(z)=e^{\int_{0}^{z} \bar{\alpha} d z}$

free electrons by ionization due to collision (in region where $\bar{\alpha}>0$ ). Where, $\bar{\alpha}$ is the effective ionization coefficient given by $\quad \bar{\alpha}(z)=\alpha(z)-\eta(z)$

$\alpha$ being Townsend's first ionization coefficient and $\eta$ the attachment coefficient [14]. Both the quantities are functions of the ambient electric field and pressure $(\mathrm{E} / \mathrm{p})$ or equivalently the ambient electric field and the number density of air $(\mathrm{E} / \mathrm{N})$. The functional dependences are reported in literature [11] and are presented in the following for reference.

$$
\begin{aligned}
& \text { For }(\mathrm{E} / \mathrm{N})>1.5 \times 10^{-15} \mathrm{~V} \mathrm{~cm}^{2} \\
& \frac{\alpha}{\mathrm{N}}=2.0 \times 10^{-16} \exp \left[\left(-7.248 \times 10^{-15}\right) /(\mathrm{E} / \mathrm{N})\right] \mathrm{cm}^{2} \\
& \text { For }(\mathrm{E} / \mathrm{N}) \leq 1.5 \times 10^{-15} \mathrm{~V} \mathrm{~cm}^{2}
\end{aligned}
$$

$$
\begin{aligned}
& \frac{\alpha}{\mathrm{N}}=6.619 \times 10^{-17} \exp \left[\left(-5.593 \times 10^{-15}\right) /(\mathrm{E} / \mathrm{N})\right] \mathrm{cm}^{2} \\
& \text { For }(\mathrm{E} / \mathrm{N})>1.05 \times 10^{-15} \mathrm{~V} \mathrm{~cm}^{2} \\
& \frac{\eta}{\mathrm{N}}=8.889 \times 10^{-5}(\mathrm{E} / \mathrm{N})+2.567 \times 10^{-19} \mathrm{~cm}^{2} \\
& \text { For }(\mathrm{E} / \mathrm{N}) \leq 1.05 \times 10^{-15} \mathrm{~V} \mathrm{~cm}^{2} \\
& \frac{\eta}{\mathrm{N}}=6.089 \times 10^{-4}(\mathrm{E} / \mathrm{N})-2.893 \times 10^{-19} \mathrm{~cm}^{2}
\end{aligned}
$$

The velocity of the moving electrons is also a function of the ambient electric field and the number density of air $(\mathrm{E} / \mathrm{N})$ and is given by [11]:

$v=2.1569 \times 10^{16}(\mathrm{E} / \mathrm{N})^{0.6064} \mathrm{~cm} / \mathrm{s}$

ii). The free electrons produced by ionizing collision will immediately attain the velocity of the avalanche head (incoming electrons) [11].

iii). The moving avalanche head can be treated as a spherical charge distribution with all the free electrons located at its head. The spherical distribution undergoes a diffusion process with the radius at any time $t$ (measured from the initiation of the avalanche) given by [17], $r=\sqrt{4 * D * t}$

where, $D$ is the diffusion coefficient for electrons.

iv) The ionization collision also produces positive space charge. Owing to their much larger mass as compared to electrons, the velocity of the space charge is orders of magnitude lower than that of the free electrons. As a result, the positive space charge can be considered to be stationary $[15,16]$.

With these routinely employed assumptions, the electromagnetic fields due to a single avalanche is studied in the following section.

\section{ELECTROMAGNETIC FIELDS DUE TO AN ELECTRON AVALANCHE}

An electron avalanche has a tiny filamentary structure in space. For measurement points located a few $\mathrm{mm}$ from the avalanche, the positive space charge can be treated as a stationary linear charge density and the spherical moving head of the avalanche as a point charge. Due to ionization and/or attachment processes as is prevalent in an avalanche, the magnitude of the point charge (avalanche head) changes with time. Therefore, the avalanche head is characterized by an arbitrarily moving point charge of time varying strength/magnitude.

\subsection{ELECTRIC FIELD DUE TO AN AVALANCHE}

The Leinard-Weichert potentials for an arbitrarily moving point charge of time varying strength are given by [12]

$$
\varphi=\frac{1}{4 \pi \varepsilon_{0}} \llbracket \frac{q(t)}{\mathcal{K} r(t)} \rrbracket
$$




$$
\vec{A}=\frac{1}{4 \pi \varepsilon_{0} c^{2}} \llbracket \frac{q(t) \vec{v}(t)}{\mathcal{K} r(t)} \rrbracket
$$

The potential for the positive space charge is given by [12]

$\varphi=\frac{1}{4 \pi \varepsilon_{0}} \int_{0}^{\llbracket R r \rrbracket} \frac{\lambda \partial l}{r}$

Where $\vec{r}, \vec{v}, \vec{a}$ are respectively the vector from measurement to source point, velocity and acceleration of the moving point charge at the retarded time, $t_{r}$.

The terms in the double square brackets represent retarded quantities.

and, $\mathcal{K}=\left(1-\frac{\hat{r} \cdot \vec{v}}{c}\right)$

Starting with the above potentials, the total electric field due to an electron avalanche can be derived [12] and is given by equation 12 .

$\vec{E}(\vec{r}, t)=\frac{\llbracket q \rrbracket}{4 \pi \varepsilon_{0} \llbracket \mathcal{K}^{3} \rrbracket} \llbracket \frac{1}{r^{2}}\left(\hat{r}-\frac{\vec{v}}{c}\right)\left(1-\frac{v^{2}}{c^{2}}\right)+\frac{1}{c r}(\hat{r} \times(\hat{r}-$ $\left.\left.\frac{\vec{v}}{c}\right) \times \frac{\vec{a}}{c}\right) \rrbracket+\frac{1}{4 \pi \varepsilon_{0}} \llbracket \frac{\partial q}{\partial t}\left(\frac{\hat{r}}{c^{2} \mathcal{K}^{2} r} \hat{v} \cdot \vec{v}-\frac{1}{c^{2} \mathcal{K}^{2}}\left(\frac{\vec{v}}{r}\right)\right) \rrbracket$

$+\frac{1}{4 \pi \varepsilon_{0}} \int_{0}^{\llbracket R r \rrbracket} \frac{\hat{r}}{r^{2}} \lambda \partial l$

$\vec{E}(\vec{r}, t)=\frac{\llbracket q \rrbracket}{4 \pi \varepsilon_{0} \llbracket \mathcal{K}^{3} \rrbracket} \llbracket \frac{1}{r^{2}}\left(\hat{r}-\frac{\vec{v}}{c}\right)\left(1-\frac{v^{2}}{c^{2}}\right)+\frac{1}{c r}(\hat{r} \times(\hat{r}-$ $\left.\left.\frac{\vec{v}}{c}\right) \times \frac{\vec{a}}{c}\right) \rrbracket+\frac{1}{4 \pi \varepsilon_{0}} \llbracket \frac{1}{c \mathcal{K}^{2} r} \frac{\partial q}{\partial t}\left(\hat{r} \times \hat{r} \times \frac{\vec{v}}{c}\right) \rrbracket+\frac{1}{4 \pi \varepsilon_{0}} \int_{0}^{\llbracket R_{r} \rrbracket} \frac{\hat{r}}{r^{2}} \lambda \partial l$

\subsection{MAGNETIC FIELD DUE TO AN AVALANCHE}

The magnetic field can be calculated from equation (9) as follows:

$\vec{B}=\nabla \times \vec{A}$

$=\frac{1}{4 \pi \varepsilon_{0} c^{2}} \nabla \times \llbracket \frac{q(t) \vec{v}(t)}{\mathcal{K} r(t)} \rrbracket$

The curl operation is to be performed on retarded quantities where the following relation is used for simplification [18].

$$
\nabla \llbracket \vec{f} \rrbracket=\llbracket \nabla \times \vec{f}-\frac{\vec{r}}{c \mathcal{K} r} \times \frac{\partial \vec{f}}{\partial t} \rrbracket
$$

where, $\vec{f}$ is a vector function.

Therefore, $\vec{B}=\nabla \times \vec{A}$

$=\frac{1}{4 \pi \varepsilon_{0} c^{2}} \llbracket \nabla \times\left(\frac{q \vec{v}}{\mathcal{K} r}\right)-\frac{\vec{r}}{c \mathcal{K} r} \times \frac{\partial}{\partial t}\left(\frac{q \vec{v}}{\mathcal{K} r}\right) \rrbracket$

The first term in the double square brackets is computed as follows.

$\nabla \times\left(\frac{q \vec{v}}{\mathcal{K} r}\right)=\left(\frac{q}{\mathcal{K} r}\right) \nabla \times \vec{v}+\nabla\left(\frac{q}{\mathcal{K} r}\right) \times \vec{v}$

$=\nabla\left(\frac{q}{\mathcal{K} r}\right) \times \vec{v}$
Since the charge i.e., source is moving, spatial derivatives w.r.t. measurement points are 0 . Therefore, $\nabla \times \vec{v}=0$

Now, $\nabla\left(\frac{q}{\mathcal{K} r}\right) \times \vec{v}=\left[\frac{1}{\mathcal{K} r} \nabla q+q \nabla\left(\frac{1}{\mathcal{K} r}\right)\right] \times \vec{v}$

$=q \nabla\left(\frac{1}{\mathcal{K} r}\right) \times \vec{v}$

Since gradient is w.r.t. measurement point which is outside the source location, $\nabla q=0$

Next, $\nabla\left(\frac{1}{\mathcal{K} r}\right)$ is computed as follows.

From equation $10, \mathcal{K}=\left(1-\frac{\hat{r} \cdot \vec{v}}{c}\right)$, therefore,

$\mathcal{K} r=\left(r-\frac{\vec{r} \cdot \vec{v}}{c}\right)$

$\nabla\left(\frac{1}{\mathcal{K} r}\right)=\left(\frac{-1}{\mathcal{K}^{2} r^{2}}\right) \nabla(\mathcal{K} r)=\left(\frac{-1}{\mathcal{K}^{2} r^{2}}\right) \nabla\left(r-\frac{\vec{r} \cdot \vec{v}}{c}\right)$

$=\left(\frac{-1}{\mathcal{K}^{2} r^{2}}\right)\left[\nabla r-\nabla\left(\frac{\vec{r} \cdot \vec{v}}{c}\right)\right]=\left(\frac{-1}{\mathcal{K}^{2} r^{2}}\right)\left(\hat{r}-\frac{\vec{v}}{c}\right)$

Since $\nabla r=\hat{r}$ and $\nabla\left(\frac{\vec{r} \cdot \vec{v}}{c}\right)=\frac{\vec{v}}{c}$

At this point, it is convenient to compute $\frac{\partial}{\partial t}\left(\frac{1}{\mathcal{K} r}\right)$ which will be required later.

$\frac{\partial}{\partial t}\left(\frac{1}{\mathcal{K} r}\right)=\left(\frac{-1}{\mathcal{K}^{2} r^{2}}\right) \frac{\partial}{\partial t}(\mathcal{K} r)=\left(\frac{-1}{\mathcal{K}^{2} r^{2}}\right) \frac{\partial}{\partial t}(\mathcal{K} r)$

$=\left(\frac{-1}{\mathcal{K}^{2} r^{2}}\right) \frac{\partial}{\partial t}\left(r-\frac{\vec{r} \cdot \vec{v}}{c}\right) \quad($ from equation 17)

$=\left(\frac{-1}{\mathcal{K}^{2} r^{2}}\right)\left[\frac{\partial r}{\partial t}-\frac{\partial}{\partial t}\left(\frac{\vec{r} \cdot \vec{v}}{c}\right)\right]$

$=\left(\frac{-1}{\mathcal{K}^{2} r^{2}}\right)\left[\frac{\partial r}{\partial t}-\frac{1}{c}\left(\vec{r} \cdot \frac{\partial \vec{v}}{\partial t}+\cdot \frac{\partial \vec{r}}{\partial t} \cdot \vec{v}\right)\right]$

Since $\vec{r}=\overrightarrow{r_{m}}-\overrightarrow{r_{s}}$

Where $\overrightarrow{r_{m}}$ is the measurement or observation point (fixed) and $\overrightarrow{r_{s}}$ is the source location at the retarded time $t_{r}$.

$\frac{\partial \vec{r}}{\partial t}=\frac{\partial \overrightarrow{r_{m}}}{\partial t}-\frac{\partial \overrightarrow{r_{s}}}{\partial t}=0-\frac{\partial \overrightarrow{r_{s}}}{\partial t}=-\vec{v}$

Also, $r=(\vec{r} \cdot \vec{r})^{1 / 2}$ therefore,

$\frac{\partial r}{\partial t}=\frac{1}{2(\vec{r} \cdot \vec{r})^{1 / 2}}\left(\vec{r} \cdot \frac{\partial \vec{r}}{\partial t}+\frac{\partial \vec{r}}{\partial t} \cdot \vec{r}\right)$

$\frac{1}{2 r}\left(2 \vec{r} \cdot \frac{\partial \vec{r}}{\partial t}\right)=\frac{1}{2 r}(-2 \vec{r} \cdot \vec{v})=-\hat{r} \cdot \vec{v}$

Using equations 22 and 23 in equation 21,

$\frac{\partial}{\partial t}\left(\frac{1}{\mathcal{K} r}\right)=\left(\frac{-1}{\mathcal{K}^{2} r^{2}}\right)\left[-\hat{r} \cdot \vec{v}-\frac{1}{c}(\vec{r} \cdot \vec{a}-\vec{v} \cdot \vec{v})\right]$

$\left(\frac{1}{\mathcal{K}^{2} r^{2}}\right)\left[\hat{r} \cdot \vec{v}+\frac{1}{c}\left(\vec{r} \cdot \vec{a}-v^{2}\right)\right]$

Using equation 20 in equation 18 ,

$\nabla\left(\frac{q}{\mathcal{K} r}\right) \times \vec{v}=q \nabla\left(\frac{1}{\mathcal{K} r}\right) \times \vec{v}=q\left(\frac{-1}{\mathcal{K}^{2} r^{2}}\right)\left(\hat{r}-\frac{\vec{v}}{c}\right) \times \vec{v}$ 


$$
\begin{aligned}
& =q\left(\frac{1}{\mathcal{K}^{2} r^{2}}\right) \vec{v} \times\left(\hat{r}-\frac{\vec{v}}{c}\right)=q\left(\frac{1}{\mathcal{K}^{2} r^{2}}\right) \vec{v} \times \hat{r} \\
& =-q\left(\frac{1}{\mathcal{K}^{2} r^{2}}\right) \hat{r} \times \vec{v}=\left(\frac{q c}{\mathcal{K}^{2} r^{2}}\right) \hat{r} \times\left(\hat{r}-\frac{\vec{v}}{c}\right)
\end{aligned}
$$

In order to obtain the second term in the double square brackets in equation 16, first the following is evaluated

$$
\begin{aligned}
& \frac{\partial}{\partial t}\left(\frac{q \vec{v}}{\mathcal{K} r}\right)=q \frac{\partial}{\partial t}\left(\frac{\vec{v}}{\mathcal{K} r}\right)+\left(\frac{\vec{v}}{\mathcal{K} r}\right) \frac{\partial q}{\partial t} \\
& =q \frac{\partial}{\partial t}\left(\frac{\vec{v}}{\mathcal{K} r}\right)+\left(\frac{\vec{v}}{\mathcal{K} r}\right) \frac{\partial q}{\partial t}=q \vec{v} \frac{\partial}{\partial t}\left(\frac{1}{\mathcal{K} r}\right)+\left(\frac{q}{\mathcal{K} r}\right) \frac{\partial \vec{v}}{\partial t}+\left(\frac{\vec{v}}{\mathcal{K} r}\right) \frac{\partial q}{\partial t} \\
& =\left(\frac{q \vec{v}}{\mathcal{K}^{2} r^{2}}\right)\left[\hat{v} \cdot \vec{v}+\frac{1}{c}\left(\vec{r} \cdot \vec{a}-v^{2}\right)\right]+\left(\frac{q}{\mathcal{K} r}\right) \vec{a}+\left(\frac{\vec{v}}{\mathcal{K} r}\right) \frac{\partial q}{\partial t}
\end{aligned}
$$

(using equation 23)

$$
\begin{aligned}
& =\left(\frac{q \vec{v}}{\mathcal{K}^{2} r^{2}}\right)\left(\hat{r} \cdot \vec{v}-\frac{v^{2}}{c}\right)+\left(\frac{q \vec{v}}{c \mathcal{K}^{2} r}\right)(\hat{r} \cdot \vec{a})+\left(\frac{q}{\mathcal{K} r}\right) \vec{a}+\left(\frac{\vec{v}}{\mathcal{K} r}\right) \frac{\partial q}{\partial t} \\
& =\left(\frac{q \vec{v}}{\mathcal{K}^{2} r^{2}}\right)\left(\hat{v}-\frac{\vec{v}}{c}\right) \cdot \vec{v}+\left(\frac{q \vec{v}}{c \mathcal{K}^{2} r}\right)(\hat{r} \cdot \vec{a})+\left(\frac{q}{\mathcal{K} r}\right) \vec{a}+\left(\frac{\vec{v}}{\mathcal{K} r}\right) \frac{\partial q}{\partial t}
\end{aligned}
$$

The second term in the double square bracket in equation 17 is given by,

$$
\begin{aligned}
& \frac{\vec{r}}{c \mathcal{K} r} \times \frac{\partial}{\partial t}\left(\frac{q \vec{v}}{\mathcal{K} r}\right) \\
& =\frac{\vec{r}}{c \mathcal{K} r} \times\left(\frac{q \vec{v}}{\mathcal{K}^{2} r^{2}}\right)\left(\hat{r}-\frac{\vec{v}}{c}\right) \cdot \vec{v}+\frac{\vec{r}}{c \mathcal{K} r} \times\left(\frac{q \vec{v}}{c \mathcal{K}^{2} r}\right)(\hat{r} \cdot \vec{a})+ \\
& \frac{\vec{r}}{c \mathcal{K} r} \times\left(\frac{q}{\mathcal{K} r}\right) \vec{a}+\frac{\vec{r}}{c \mathcal{K} r} \times\left(\frac{\vec{v}}{\mathcal{K} r}\right) \frac{\partial q}{\partial t} \\
& =-\left(\frac{q}{\mathcal{K}^{3} r^{2}}\right) \hat{r} \times\left(\hat{r}-\frac{\vec{v}}{c}\right)\left(\hat{r}-\frac{\vec{v}}{c}\right) \cdot \vec{v}+(\hat{r} \cdot \vec{a}) \hat{r} \times \\
& \left(\frac{q \vec{v}}{c^{2} \mathcal{K}^{3} r}\right)+\left(\frac{q}{c \mathcal{K}^{2} r}\right) \hat{r} \times \vec{a}+\frac{\partial q}{\partial t} \frac{1}{\mathcal{K}^{2} r} \hat{r} \times\left(\frac{\vec{v}}{c}\right) \\
& =-\left(\frac{q}{\mathcal{K}^{3} r^{2}}\right) \hat{r} \times\left(\hat{r}-\frac{\vec{v}}{c}\right)\left(\hat{r}-\frac{\vec{v}}{c}\right) \cdot \vec{v}+\left(\frac{q}{c \mathcal{K}^{3} r}\right) \hat{r} \times \\
& \left((\hat{r} \cdot \vec{a}) \frac{\vec{v}}{c}+\mathcal{K} \vec{a}\right)+\frac{\partial q}{\partial t} \frac{1}{\mathcal{K}^{2} r} \hat{\sim} \times\left(\frac{\vec{v}}{c}\right) \\
& =-\left(\frac{q}{\mathcal{K}^{3} r^{2}}\right) \hat{r} \times\left(\hat{r}-\frac{\vec{v}}{c}\right)\left(\hat{r}-\frac{\vec{v}}{c}\right) \cdot \vec{v}+\left(\frac{q}{c \mathcal{K}^{3} r}\right) \hat{r} \times \\
& \left((\hat{r} \cdot \vec{a}) \frac{\vec{v}}{c}+\left(\hat{r} \cdot \hat{r}-\frac{\hat{r} \cdot \vec{v}}{c}\right) \vec{a}\right)+\frac{\partial q}{\partial t} \frac{1}{\mathcal{K}^{2} r} \hat{r} \times\left(\frac{\vec{v}}{c}\right) \\
& =-\left(\frac{q}{\mathcal{K}^{3} r^{2}}\right) \hat{r} \times\left(\hat{r}-\frac{\vec{v}}{c}\right)\left(\hat{r}-\frac{\vec{v}}{c}\right) \cdot \vec{v}-\left(\frac{q}{c \mathcal{K}^{3} r}\right) \hat{r} \times \\
& \left((\hat{r} \cdot \vec{a})\left(\hat{r}-\frac{\vec{v}}{c}\right)-\hat{r} \cdot\left(\hat{r}-\frac{\vec{v}}{c}\right) \vec{a}\right)-\frac{\partial q}{\partial t} \frac{1}{\mathcal{K}^{2} r} \hat{r} \times\left(\hat{r}-\frac{\vec{v}}{c}\right) \\
& =-\left(\frac{q}{\mathcal{K}^{3} r^{2}}\right) \hat{r} \times\left(\hat{r}-\frac{\vec{v}}{c}\right)\left(\hat{r}-\frac{\vec{v}}{c}\right) \cdot \vec{v}-\left(\frac{q}{c \mathcal{K}^{3} r}\right) \hat{r} \times \\
& \left((\hat{r} \cdot \vec{a})\left(\hat{r}-\frac{\vec{v}}{c}\right)-\hat{r} \cdot\left(\hat{r}-\frac{\vec{v}}{c}\right) \vec{a}\right)-\frac{\partial q}{\partial t} \frac{1}{\mathcal{K}^{2} r} \hat{r} \times\left(\hat{r}-\frac{\vec{v}}{c}\right) \\
& =-\left(\frac{q}{\mathcal{K}^{3} r^{2}}\right) \hat{r} \times\left(\hat{r}-\frac{\vec{v}}{c}\right)\left(\hat{r}-\frac{\vec{v}}{c}\right) \cdot \vec{v}-\left(\frac{q}{\mathcal{K}^{3} r}\right) \hat{r} \times(\hat{r}- \\
& \left.\frac{\vec{v}}{c}\right) \times \frac{\vec{a}}{c}-\frac{\partial q}{\partial t} \frac{1}{\mathcal{K}^{2} r} \hat{r} \times\left(\hat{r}-\frac{\vec{v}}{c}\right)
\end{aligned}
$$

From equations 17, 25 and 27, the magnetic field can be evaluated as,

$$
\begin{aligned}
& \vec{B}=\frac{1}{4 \pi \varepsilon_{0} c^{2}} \llbracket\left(\frac{q c}{\mathcal{K}^{2} r^{2}}\right) \hat{r} \times\left(\hat{r}-\frac{\vec{v}}{c}\right)+\left(\frac{q}{\mathcal{K}^{3} r^{2}}\right) \hat{r} \times(\hat{r}- \\
& \left.\frac{\vec{v}}{c}\right)\left(\hat{r}-\frac{\vec{v}}{c}\right) \cdot \vec{v}+\left(\frac{q}{\mathcal{K}^{3} r}\right) \hat{r} \times\left(\hat{r}-\frac{\vec{v}}{c}\right) \times \frac{\vec{a}}{c}+\frac{\partial q}{\partial t} \frac{1}{\mathcal{K}^{2} r} \hat{r} \times \\
& \left(\hat{r}-\frac{\vec{v}}{c}\right) \rrbracket \\
& =\frac{1}{4 \pi \varepsilon_{0} c^{2}} \llbracket\left(\frac{q c}{\mathcal{K}^{3} r^{2}}\right) \hat{r} \times\left(\hat{r}-\frac{\vec{v}}{c}\right)\left(\mathcal{K}+\left(\hat{r}-\frac{\vec{v}}{c}\right) \cdot \frac{\vec{v}}{c}\right)+ \\
& \left(\frac{q}{\mathcal{K}^{3} r}\right) \hat{r} \times\left(\hat{r}-\frac{\vec{v}}{c}\right) \times \frac{\vec{a}}{c}+\frac{\partial q}{\partial t} \frac{1}{\mathcal{K}^{2} r} \hat{r} \times\left(\hat{r}-\frac{\vec{v}}{c}\right) \rrbracket \\
& =\frac{1}{4 \pi \varepsilon_{0} c^{2}} \llbracket\left(\frac{q c}{\mathcal{K}^{3} r^{2}}\right) \hat{r} \times\left(\hat{r}-\frac{\vec{v}}{c}\right)\left(1-\frac{v^{2}}{c^{2}}\right)+\left(\frac{q}{\mathcal{K}^{3} r}\right) \hat{r} \times \\
& \left(\hat{r}-\frac{\vec{v}}{c}\right) \times \frac{\vec{a}}{c}+\frac{1}{\mathcal{K}^{2} r} \frac{\partial q}{\partial t} \hat{r} \times\left(\hat{r}-\frac{\vec{v}}{c}\right) \rrbracket
\end{aligned}
$$

This is the general expression for the Magnetic field due to an electron avalanche. The first term in the expression varies as $\frac{1}{r^{2}}$ and therefore is the near field component of the magnetic field. The remaining two terms fall as $\frac{1}{r}$ and hence are the far field or radiation components. The separable form of the different terms makes it convenient and simple to evaluate the radiation and wave propagation effects which will be carried out in section 5 .

\section{VALIDATION OF THE EXPRESSION FOR MAGNETIC FIELD}

The derived expression for the magnetic field (equation 28) needs to be validated. Since the electric field given by equations 12 (or 13) has been validated already in [12], validation of the magnetic field can be performed by using Maxwell's curl equation for the electric field i.e., Faraday's law.

$$
\begin{aligned}
& \nabla \times \vec{E}=-\frac{\partial \vec{B}}{\partial t} \\
& \text { or, } \vec{B}=-\int_{0}^{t}(\nabla \times \vec{E}) \partial t
\end{aligned}
$$

Equation 30 is solved numerically and is compared with the solution obtained from equation 28 for validation.

The validation exercise has been carried out for avalanches developing under different background electric fields. However, sample results are presented for the exponentially decreasing (with distance) field as shown in figure 1.

An electron avalanche developing under the electric field shown in figure 1 has been studied and traced in literature [19], thereby providing a reference. In accordance with the study carried out in literature [19], the avalanche has been assumed to grow along a straight line, assumed as the z-axis, in the present work. The point of initiation of the avalanche is assumed to be the origin without loss of generality. 


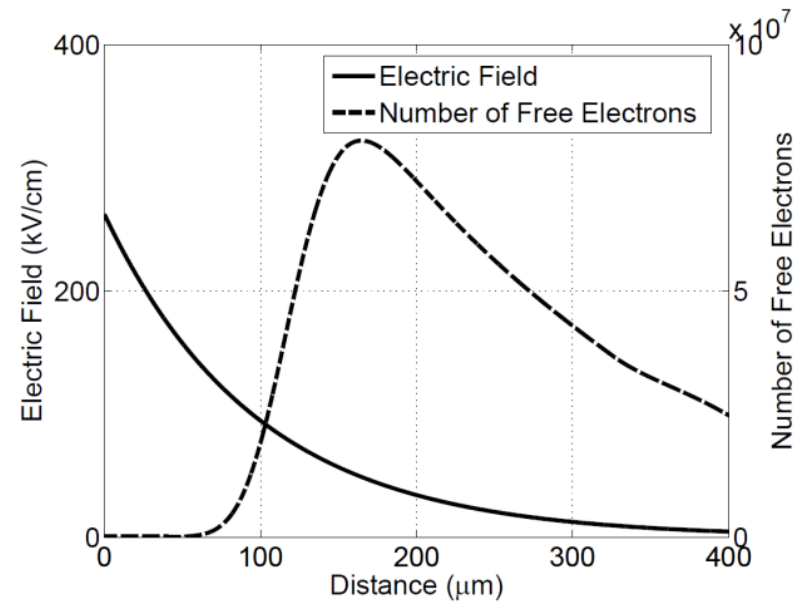

Figure 1. Spatial variation of electric field for tracing electron avalanche and number of free electrons in the electron avalanche

The number of free electrons produced by ionization as the avalanche develops in space is computed using equation 1 with the coefficients obtained from equations 2 to 5 . The variation is also shown in figure 1 .

The magnetic field produced by the electron avalanche traced above is computed at different measurement points using:

(a) the analytical expression derived in equation 28 .

(b) Faraday's law equation 30

The results are compared. Sample results are presented in figures 2 and 3. For an avalanche growing along the $z$-axis, the $z$-component of the magnetic field is identically zero for all time. Therefore, only the $x$ and $y$-components of the fields are shown in the following.

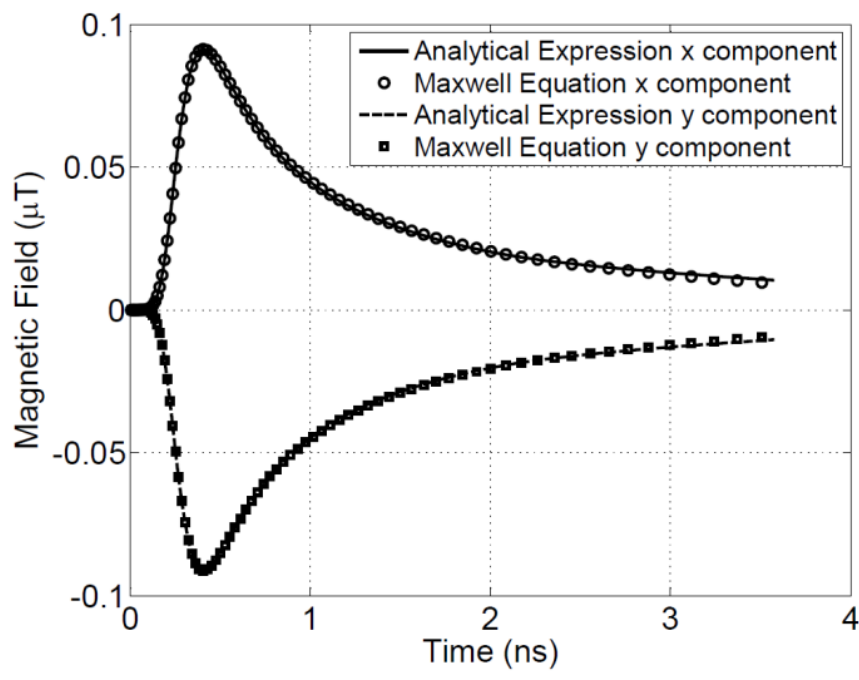

Figure 2. Magnetic field at $(0.25 \mathrm{~mm}, 0.25 \mathrm{~mm}, 1 \mathrm{~mm})$ due to electron avalanche
The magnetic field at $(0.25 \mathrm{~mm}, 0.25 \mathrm{~mm}, 1 \mathrm{~mm})$ versus time has been shown in figure 2 . The result of the analytical expression is found to be in excellent agreement with that obtained by the numerical solution of Faraday's law. As the avalanche is considered to initiate at the origin, the observation point is located very close to the avalanche (approximately $1.1 \mathrm{~mm}$ ). At such distances, the near field component (first term in equation 28) dominates over the far or radiation components (approximately 50 times in the present case).

The opposite scenario i.e., distance at which the far or radiation field dominates over the near field has been shown in figure 3 . The field given by the analytical expression agrees excellently with that obtained by the numerical solution of Faraday's law at $(1 \mathrm{~m}, 1 \mathrm{~m}, 0.001 \mathrm{~m})$.

The observation point being located approximately $1.414 \mathrm{~m}$ from the avalanche, the radiation component of the field dominates over the near field term (by approximately 30 times) in the present case. In figure 3, the retardation effect is also clearly observed. The field remains zero till approximately 4.7ns. This is equal to the time taken for the field to reach the measurement point from the avalanche location (1.414m away).

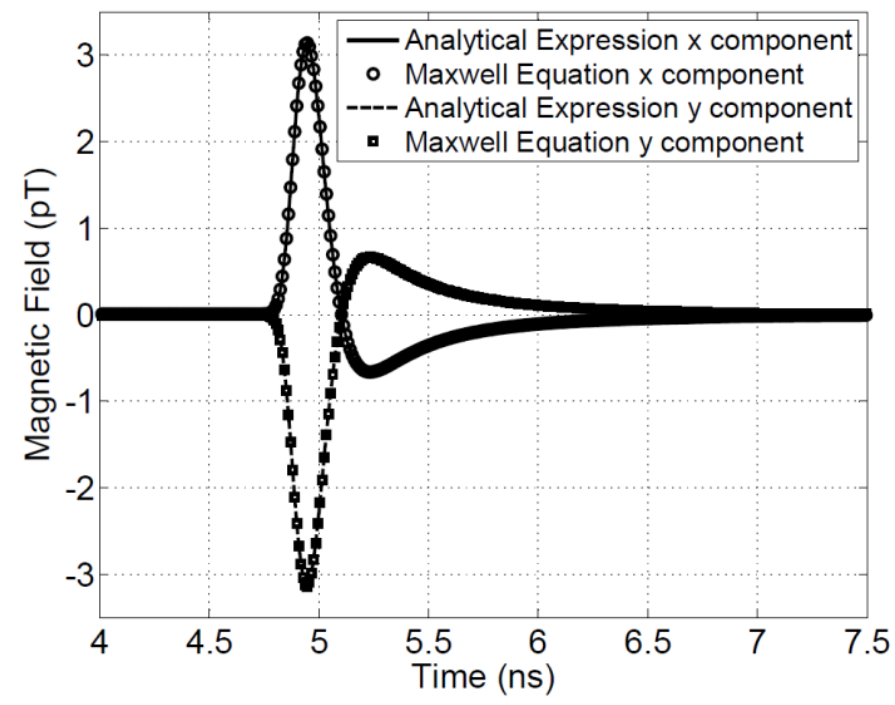

Figure 3. Magnetic field at $(1 \mathrm{~m}, 1 \mathrm{~m}, 0.001 \mathrm{~m})$ due to electron avalanche

The sample results presented in this section have demonstrated that the analytical expression for the magnetic field derived in the present work is in complete agreement with the solution of Maxwell's equation (numerical). This is true for the near field region, far field region as well as regions where the near and far fields are comparable in magnitude. The above exercise therefore provides a validation for the derived expression (equation 28) for the magnetic field due to an electron avalanche.

In deriving equation 28, no assumption has been made with regards to the velocity of the avalanche head (moving electrons). In other words, not only can the avalanche develop along any arbitrary path, but it can also attain speeds 
comparable to the speed of light. In the following, a brief discussion is carried out about relativistic electron avalanches.

\section{RELATIVISTIC ELECTRON AVALANCHE}

Relativistic avalanches have been observed to occur in nature [13]. Since the derived expression for magnetic field (equation 28) is valid for any physical speed which the charges can attain, it must be valid for relativistic avalanches as well. In the following, sample results are presented to demonstrate the same.

Although the Townsend coefficients ( $\alpha$ and $\eta$ ) given by equations 2 to 5 and $v$ (equation 6) are not obtained under relativistic conditions, it is assumed that they are valid for relativistic avalanches as well. In addition, the velocity is scaled up to relativistic levels.

In the validation exercise, the results of equation 28 and 30 are compared. Since the same aforesaid assumptions are applied to both the equations, the validation exercise holds.

Sample results shown in the following is for an avalanche which has the same spatial variation of the number of free electrons as in figure 1, but with the velocity scaled up by 400 times. The magnitude of maximum velocity of the avalanche head is $94 \%$ the speed of light for this case. The $x$ and $y$ components of the magnetic field produced by this relativistic avalanche at the observation point $(0.5 \mathrm{~cm}, 0.75 \mathrm{~cm}, 1 \mathrm{~cm})$ is shown in figure 4.

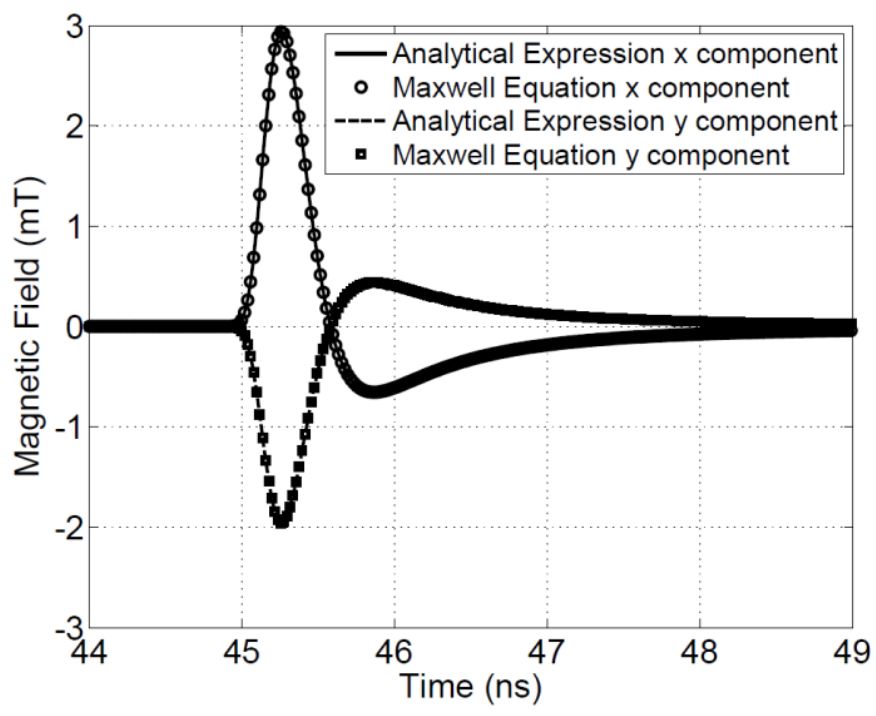

Figure 4. Magnetic field at $(0.5 \mathrm{~cm}, 0.75 \mathrm{~cm}, 1 \mathrm{~cm})$ due to a relativistic electron avalanche

The above exercise demonstrates that for relativistic electron avalanche, the results of the analytical expression agree completely with the numerical solution of Maxwell's equation. Therefore, the expression derived is valid for all physical velocities which the avalanche head can achieve i.e., from 0 to velocities comparable to the speed of light.

\section{CONCLUSIONS}

In power system and electronic circuits, local partial breakdown in the form of corona, PD and ESD are common. The electromagnetic fields due to these processes can lead to EMI, may cause damage or might be useful in detecting and locating internal discharges. It is therefore important to relate the electromagnetic fields produced, to the physical ionization process constituting the discharge. As a first step, the electric field due to the basic ionization process of an electron avalanche has been derived and validated in literature. The complete expression for the magnetic field has been derived in the present work. The derived expression provides the near field and far or radiation field terms separately, making it possible to study them individually. It has been shown to be in complete agreement with the solution of Maxwell's equation (numerical) at various distances and even for relativistic avalanches. Further, a simplified approach proposed in literature to estimate the radiation component of electric field due to discharge has been applied for the present case of magnetic field. It has been demonstrated that the upper frequency range and the field levels (peak magnitude) due to discharge can be estimated to good accuracy using results of single electron avalanche. Such an approach could be useful in EMI/EMC studies, designing protection circuits against EM fields or selecting/designing suitable measurement and calibration systems.

To conclude, the present work has been able to relate the magnetic field produced to the basic physical ionization process and derive a general expression for the same starting from the fundamental retarded scalar and vector potentials (LeinardWeichert potentials).

\section{REFERENCES}

[1] V. Javor, K. Lundengård,M. Rančić and S. Silvestrov, "A Study on Frequency Spectrum of Electrostatic Discharge Currents and Lightning Currents" In 2019 14th International Conference on Advanced Technologies, Systems and Services in Telecommunications (TELSIKS), 2019, (pp. 198-201). IEEE.

[2] J. Lee, Y. Huh, P. Bendix and S. M. Kang, "Design of ESD power protection with diode structures for mixed-power supply systems". IEEE Journal of Solid-State Circuits, 39(1), pp.260-264, 2004

[3] Report, I.R.N.S., "Comparison of radio noise prediction methods with CIGRE/IEEE survey results". IEEE Transactions on Power Apparatus and Systems, (3), pp.1029-1042, 1973.

[4] J.J. LaForest, Transmission-line reference book. $345 \mathrm{kV}$ and above (No. EPRI-EL-2500). General Electric Co., Pittsfield, MA (USA). Large Transformer Div.; General Electric Co., Schenectady, NY (USA). Electric Utility Systems Engineering Dept., 1981.

[5] EPRI, A., Transmission Line Reference Book-200 kV and Above. Electric Power Research Institute, 2005.

[6] A. Darwish, S.S. Refaat, H.A. Toliyat, and H. Abu-Rub, "On the electromagnetic wave behavior due to partial discharge in gas insulated switchgears: State-of-art review”, IEEE Access, 7, pp.75822-75836, 2019

[7] M.K. Chen, J.M. Chen, and C.Y. Cheng, "Partial discharge detection by RF coil in $161 \mathrm{kV}$ Power Transformer", IEEE Transactions on Dielectrics and Electrical Insulation, 21(3), pp.1405-1414, 2014

[8] Z. Tang, C. Li, X. Cheng, W. Wang, J. Li and J. Li, "Partial discharge location in power transformers using wideband RF detection", IEEE Transactions on Dielectrics and Electrical Insulation, 13(6), pp.11931199, 2006.

[9] J. Rubio-Serrano, M.V. Rojas-Moreno, J. Posada, J.M. Martínez-Tarifa, G. Robles and J.A. Garcia-Souto, "Electro-acoustic detection, identification 
and location of partial discharge sources in oil-paper insulation systems", IEEE Transactions on Dielectrics and Electrical Insulation, 19(5), pp.1569-1578, 2012

[10] O. Beniugă, A. Sălceanu, O. Neacşu and K. Kováč, "Time domain measurement of magnetic field radiated by electrostatic discharge for electromagnetic pollution assessment" In 2012 International Conference and Exposition on Electrical and Power Engineering (pp. 632-635). IEEE.

[11] V. Cooray and G. Cooray, "Electromagnetic radiation field of an electron avalanche". Atmospheric research, 117, pp.18-27. 2012.

[12] D. Nath and U. Kumar, "Total electric field due to an isolated electron avalanche", IEEE Transactions on Dielectrics and Electrical Insulation, 23(5), pp.2562-2571, 2016.

[13] V. Cooray, T. Marshall, G. Cooray, J. Dwyer and S. Arabshahi, "Electric field of a relativistic electron avalanche", In 2012 International Conference on Lightning Protection (ICLP) (pp. 1-5). IEEE.

[14] L.B. Loeb, Electrical Coronas: their Basic Physical Mechanisms, University of California press, 1965.

[15] P.S. Maruvada, Corona Performance of High-Voltage Transmission Lines, Overseas press, 2000.

[16] E. Nasser, Fundamentals of gaseous ionization and plasma electronics. John Wiley \& Sons, 1971

[17] H. Parekh and K. D. Srivastava. "Effect of avalanche space charge field on the calculation of corona onset voltage", IEEE Trans. Electr. Insul., Vol. 4, pp.181-192, 1979.

[18] J.D. Jackson, Classical electrodynamics third edition. Wiley, 1999.

[19] V. Cooray, Lightning Electromagnetics, IET Digital Library, 2012

[20] U. Kumar and D. Nath, "Measurement of electron avalanche current in a point-plane geometry stressed with negative voltage", In 2015 International Conference on Condition Assessment Techniques in Electrical Systems (CATCON) (pp. 247-251). IEEE. 